\title{
Scattering Effects in a Positron Lifetime Beam Line
}

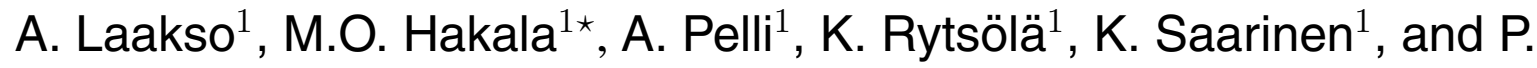 \\ Hautojärvi ${ }^{1}$
}

Laboratory of Physics, Helsinki University of Technology, antti.laakso @ hut.fi

P.O. Box 1100, FIN02015 HUT, Finland

Keywords: Positron spectroscopy, lifetime beam, electron scattering, simulations

\begin{abstract}
Electrons can be used as test particles of the pulsed positron lifetime beam. The beam should be designed so that electron scattering effects do not distort the timing spectrum. To study these effects we have developed a simulation program which takes into account the collisions of the particles with beam structures and calculates the final time distribution. This program has been used in the design of an improved chopper for positron lifetime beam.
\end{abstract}

\section{Introduction}

To test the properties of a pulsed positron lifetime beam electrons can be used as test particles [1]. Electrons have many advantages over positrons, eg. an easily variable intensity and a narrow, welldefined beam spot. The scattering effects are a significant aspect that has to be taken into account both with electrons and positrons. The electrons colliding with the beam pulsing structures can scatter in the forward direction or create secondary electrons, producing electrons with lower energy than the initial particle. These electrons increase the background and the width of the final time spectrum. This problem can be avoided by proper mechanical design of the pulsing components in the beam.

To study these effects we have developed a simulation program that calculates the trajectories of the electrons (or positrons) and the time distribution at the end of the beam. The essential part of this simulation is the proper treatment of the collision process of the particles. We have studied this process in detail with direct Monte Carlo simulations, which take into account the scattered and secondary electrons. The data from the simulations have been used to create a model that has been applied to calculate the trajectories and the time distribution of the electrons (and which is also applicable to positrons). We present the results of the simulations and compare the data with the experimental results.

\section{Setup and Methods}

The pulsed positron lifetime beam The concept of the pulsed positron lifetime beam at Helsinki University of Technology is described in detail in [2]. The source, velocity selector and the beam bunching and chopping stages float at high voltage whereas the sample is at ground potential. The source area comprises a ${ }^{22} \mathrm{Na}$ source followed by a W-moderator. Slow positrons are separated from fast ones in a $90^{\circ}$ bend in the beam. The first pulsing stage, an unmatched double gap buncher, is the prebuncher followed by a drift tube and a pre-accelerator where positrons reach an energy of $1 \mathrm{keV}$. After this acceleration the beam is chopped in a deflection chopper shown Fig.1. When the deflecting field differs from zero the trajectories of the positrons bend and they collide with the walls of the chopper. The chopped beam is then bunched in the main buncher. The final acceleration is done with

\footnotetext{
* Present address: Division of X-ray Physics, Department of Physical Sciences

P.O.Box 64, FIN-00014, University of Helsinki, Finland
} 
an accelerator-decelerator stage. When the system is tested with electrons the source is replaced with an electron gun and a microchannel plate is used as the detector.

The chopper The chopper, shown in Fig.1, is composed of two copper blocks and a plate in between of them. A deflection electrode is placed at the entrance of the chopper. The deflected positrons collide with the walls of the chopper and either annihilate or scatter. When the electrons with energy of $1 \mathrm{keV}$ collide with chopper walls they either scatter or create secondary electrons both with energies less than the initial energy. The scattered positrons or electrons as well as the secondary electrons can traverse through the

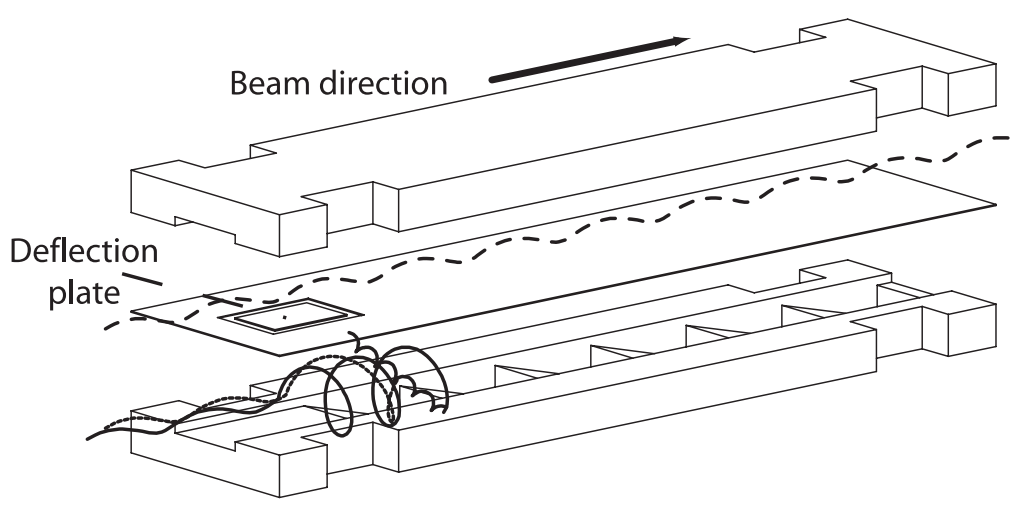

Fig. 1. Schematical drawing of the chopper with a sawtooth structure preventing scatterings in the forward direction. Electrons (positrons) can pass through, scatter and create secondary electrons (or annihilate).

chopper and contribute to the final timing spectrum if the chopper geometry is not properly chosen.

\section{The simulations}

Monte-Carlo simulation of the scattering process. In order to properly model the scattering process of electrons hitting the wall, we have studied the scattering and the secondary electron emission processes with Monte Carlo simulations [3, 4]. In these simulations the trajectories of the primary (PE) and the created secondary (SE) electrons are followed one by one inside the solid until they re-emit from the surface. The relevant inelastic and elastic scattering cross sections are used to stochastically sample the type of the collision (elastic/inelastic), path length $\lambda$ between the collisions, energy and momentum transfer $(\omega, q)$, angular deflection in a collision, and the excitation probability of a SE.

The simulations give the outgoing electron energy distribution as a function of the energy and angle of the incident electron, allowing the calculation of the energy loss of the scattered primary electrons and the yield of the secondary electrons. In the practical simulation, we have calculated the probability distribution for the outgoing electron current for various energies and angles of the incident primary electron, and these are used to determine stochastically the energy and angle of the emitted electrons. An example of this is shown in Fig. 2

Trajectory calculation and parametrization of the Monte Carlo data. The electron trajectories $\mathbf{r}(t)$ were calculated, based on the initial position and velocity distribution, using classical equations of motion for a particle in a $\mathbf{E}$ - and B-field:

$$
q \mathbf{E}_{\|}(\mathbf{r})+q \mathbf{E}_{\perp}(\mathbf{r}, t)+q \frac{d \mathbf{r}(t)}{d t} \times \mathbf{B}(\mathbf{r})=m \frac{d^{2} \mathbf{r}(t)}{d t^{2}}
$$

The guiding axial magnetic field $\mathbf{B}(\mathbf{r})$ was assumed to be uniform and the time varying electric field of the chopper $\mathbf{E}_{\perp}(\mathbf{r}, t)$ and the accelerating fields $\mathbf{E}_{\|}(\mathbf{r})$ along the beam line were taken into account in each time and place step. In case of collision with walls or electrodes the routine taking care of scatterings was employed.

To reduce calculation time of electron trajectories the numerical data obtained in MC simulations were parametrized (Fig. 2) to generate analytical expressions for electron emission distributions for different incoming particle angles and energies. It should be noted that using this approach to treat the scattering effects renders primary and secondary electrons indistinguishable in the simulation data. 

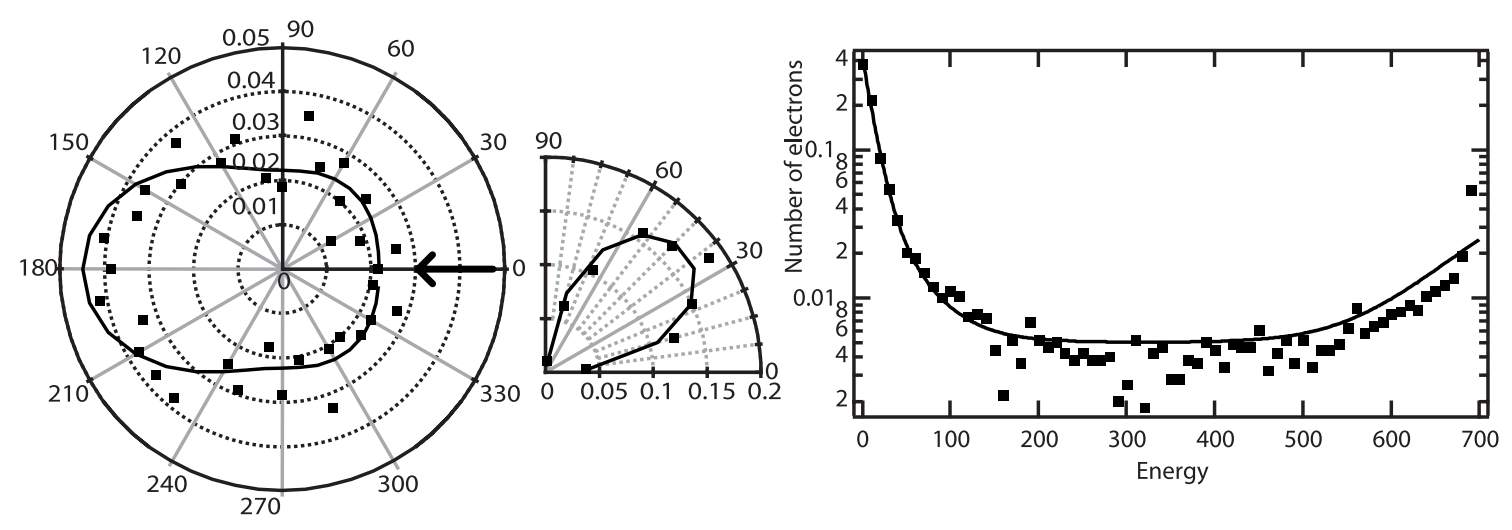

Fig. 2. An example of angular and energy distributions for emitted electrons: left - azimuthal, middle - polar, right - energy. The direction of the incoming electron is marked with an arrow. The simulated values are shown with squares and the parametrized distribution is shown with solid line. The incoming polar angle was $20^{\circ}$ and energy $700 \mathrm{eV}$.

The time distribution was created at the end of the beam by calculating in which phase of the pulsing period the electrons arrive and histogramming these times.

\section{Results}

To test the simulation code two different chopper geometries were studied and the results were compared with experimental data. The chopper geometries taken into investigation were the flat chopper and the "sawtooth" chopper, the walls of which are grooved so that the probability for forward scattering is minimal. The compari-

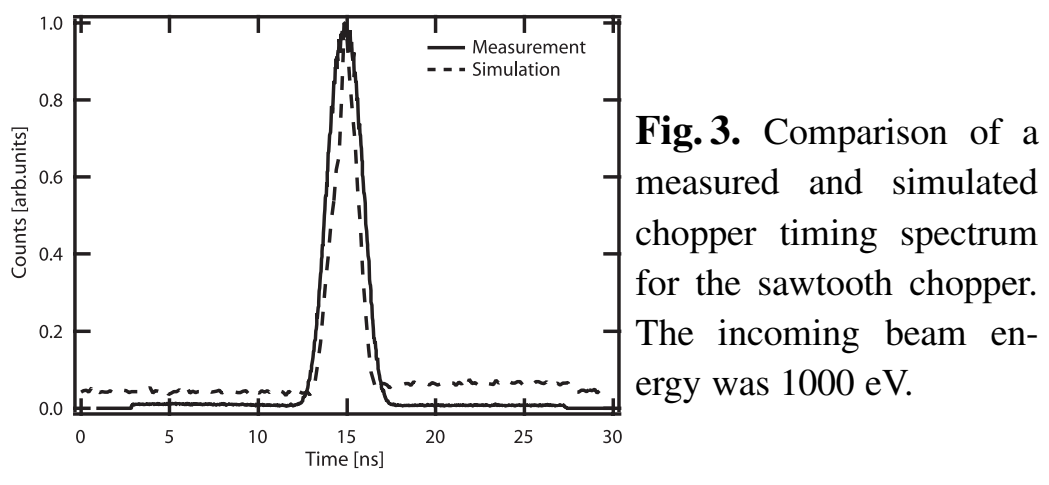
son of the simulated and the experimental data for the "sawtooth" chopper is shown in Fig. 3. As a result we can say that the simulated spectrum correspond relatively well with the measured one.

\section{Conclusions and discussion}

We have developed a simulation program to study electron scattering effects that are present when a pulsed positron lifetime beam is tested with electrons. The results from the simulations agree with experimental data showing that the simulation program can be used in the design of the pulsed beam. In addition, the simulation can be easily adopted to study positron scattering effects in a pulsed positron beam by doing MC simulations for the collisions of positrons and using this data for trajectory and time distribution calculations.

\section{References}

[1] A. Laakso, A. Pelli, K. Rytsölä, K. Saarinen, P. Hautojärvi, unpublished, (2003).

[2] K. Fallström, T. Laine, Appl. Surf. Sci., Vol. 149 (1999) pp. 44 -48.

[3] M. Hakala, C. Corbel, R. M. Nieminen, unpublished, (2003).

[4] S. Valkealahti, R. Nieminen, Applied-Physics-A-(Solids-and-Surfaces), Vol. A35 (1) (1984) pp. 51 -59. 